\title{
Efficient silicon integrated four-mode edge coupler for few-mode fiber coupling
}

\author{
Junbo Zhu (朱俊波 $)^{1,2, \dagger}$, Haiyang Huang (黄海阳) $)^{1, \dagger}$, Yingxuan Zhao (赵瑛璇) ${ }^{1}$, Yang Li (李 杨 $)^{1,2}$, Zhen Sheng (盛 振 $)^{1 *}$, and \\ Fuwan Gan (甘甫烷) $)^{1,3^{* *}}$ \\ ${ }^{1}$ State Key Laboratory of Functional Materials for Informatics, Shanghai Institute of Microsystem and Information Technology, Chinese Academy of \\ Sciences, Shanghai 200050, China \\ ${ }^{2}$ University of Chinese Academy of Sciences, Beijing 100049, China \\ ${ }^{3}$ School of Future Technology, University of Chinese Academy of Sciences, Beijing 100049, China
}

*Corresponding author: zsheng@mail.sim.ac.cn

${ }^{*}$ Corresponding author: fuwan@mail.sim.ac.cn

Received April 29, 2021 | Accepted July 30, 2021 | Posted Online September 22, 2021

\begin{abstract}
Here, we designed a broadband, low loss, compact, and fabrication-tolerant silicon-based four-mode edge coupler, composed of a $1 \times 3$ adiabatic mode-evolution counter-taper splitter and a triple-tip inverse taper. Based on mode conversion and power splitting, the proposed structure can simultaneously realize efficient mode coupling from $T_{0}, T M_{0}, T E_{1}$, and $T M_{1}$ modes of multimode silicon waveguides to linearly polarized (LP), $L P_{01, x}, L P_{01, y}, L P_{11 a, x}$ and $L P_{11 a, y}$, modes in the few-mode fiber. To the best of our knowledge, we proposed the first scheme of four LP modes coupling, which is fully compatible with standard fabrication process. The 3D finite-difference time-domain simulation results show that the on-chip conversion losses of the four modes remain lower than $0.62 \mathrm{~dB}$ over the $200 \mathrm{~nm}$ wavelength range, and total coupling losses are $4.1 \mathrm{~dB}$, $5.1 \mathrm{~dB}, 2.1 \mathrm{~dB}$, and $2.9 \mathrm{~dB}$ for $T E_{0}$-to- $L P_{01, x}, T M_{0}-t_{0}-L_{P_{01, y}}, T E_{1}-t_{0}-L_{P_{11 a, x}}$ and $T M_{1}$-to- $L P_{11 a, y}$, respectively. Good fabrication tolerance and relaxed critical dimensions make the four-mode edge coupler compatible with standard fabrication process of commercial silicon photonic foundries.
\end{abstract}

Keywords: multimode coupling; CMOS compatibility; silicon waveguide; few-mode fiber.

DOI: 10.3788/COL202220.011302

\section{Introduction}

Silicon photonics has been attracting lots of attention in recent years ${ }^{[1]}$, benefiting from the compatibility with standard complementary metal-oxide-semiconductor (CMOS) fabrication technology, which significantly reduces the fabrication cost of silicon photonic devices. The silicon-on-insulator (SOI) platform has been widely used in on-chip communication systems with low insertion loss and small footprint ${ }^{[2,3]}$. Meanwhile, in order to satisfy the rapidly increasing demands on high capacity and low power consumption data transfer, mode-division multiplexing (MDM) has also emerged as an efficient approach to further increase the transmission capability ${ }^{[4]}$ with the introduction of high-order modes in both fibers and silicon chips ${ }^{[5,6]}$. Many on-chip multichannel mode (de)multiplexers have been developed $^{[7-9]}$, but, in order to implement a complete on-chip MDM optical network, large data transmission capacity and low-loss coupling between few-mode fibers (FMFs) and on-chip multimode waveguide are also highly needed ${ }^{[10]}$.
It is a challenging task to design efficient couplers with large bandwidths, low insertion loss, and small footprint that connect on-chip multimode waveguides and FMFs due to their huge mode mismatch. Vertical and edge coupling schemes are the most common ways to realize multimode fiber-chip coupling. For vertical coupling, grating couplers (GCs) ${ }^{[11-14]}$ are usually used due to their small footprint and large misalignment tolerance. However, the GCs are intrinsically sensitive to fabrication error and wavelength, which significantly limit their application in broadband wavelength-division multiplexing (WDM). Compared with GCs, edge couplers can overcome the drawbacks of polarization/wavelength sensitivity. The 3D-mode multiplexer structure based on multilayer asymmetric waveguide branches has been reported for four-mode coupling ${ }^{[15]}$, while the fabrication becomes complicated for different waveguide heights. Multi-stage silicon inverse taper couplers with polymer up-cladding realized four linearly polarized (LP) modes coupling $^{[16]}$, but the large total length $(2.68 \mathrm{~mm})$ and lack of compatibility with standard CMOS fabrication process limited its application. The double-tip inverse taper was demonstrated to 
realize the first, to the best of our knowledge, high-order mode coupling $^{[17]}$, and some three inverse tapers for two-mode coupling have been reported ${ }^{[10,18]}$ with large mode conversion losses and coupling losses ${ }^{[10]}$. Although these types of couplers mentioned can meet some performance requirements, it is a challenge for an on-chip multimode coupler to simultaneously satisfy compact, low loss, wavelength-independent, and CMOS compatibility.

In this Letter, we report a silicon edge coupler based on adiabatic taper for four-mode fiber-to-chip coupling. The proposed coupler consists of a $1 \times 3$ adiabatic mode-evolution countertaper splitter and a triple-tip inverse taper. The 3D finitedifference time-domain (FDTD) simulation results show that the on-chip conversion losses of the four-mode edge coupler (FMEC) are $0.01 \mathrm{~dB}, 0.02 \mathrm{~dB}, 0.07 \mathrm{~dB}$, and $0.27 \mathrm{~dB}$ for the input $\mathrm{TE}_{0}, \mathrm{TM}_{0}, \mathrm{TE}_{1}$, and $\mathrm{TM}_{1}$ modes at $1.55 \mu \mathrm{m}$ wavelength, respectively, and, in the wavelength range from 1.45 to $1.65 \mu \mathrm{m}$, the mode conversion loss remains lower than $0.62 \mathrm{~dB}$. Meanwhile, the designed feature size is $100 \mathrm{~nm}$, which is compatible with the standard process of commercial silicon photonic foundries. The calculated total coupling losses between the edge coupler and FWF are $4.1 \mathrm{~dB}, 5.1 \mathrm{~dB}, 2.1 \mathrm{~dB}$, and $2.9 \mathrm{~dB}$ for $\mathrm{TE}_{0}$-to- $\mathrm{LP}_{01, \mathrm{x}}$, $\mathrm{TM}_{0}$-to- $\mathrm{LP}_{01, \mathrm{y}}, \mathrm{TE}_{1}$-to- $\mathrm{LP}_{11 \mathrm{a}, \mathrm{x}}$, and $\mathrm{TM}_{1}$-to- $\mathrm{LP}_{11 \mathrm{a}, \mathrm{y}}$, respectively. Moreover, the fabrication tolerance analysis confirms that the FMEC remains fairly low loss within a large deviation range ( $\pm 50 \mathrm{~nm}$ for silicon waveguide width).

\section{Operation Principle and Device Design}

Figure 1(a) presents the schematic of the designed FMEC, which consists of a $1 \times 3$ adiabatic mode-evolution counter-taper splitter and a triple-tip inverse taper. The FMEC is designed on an SOI wafer with $220 \mathrm{~nm}$ thick top silicon layer, $4.5 \mu \mathrm{m}$ thick upper $\mathrm{SiO}_{2}$ cladding, and $3 \mu \mathrm{m}$ thick bottom $\mathrm{SiO}_{2}$ cladding. The operation principle in the FMEC is based on the mode evolution in a coupler that has two waveguides with cores countertapered ${ }^{[19,20]}$. As shown in Fig. 1(b), the width of the upper waveguide (WG1) increases from 0.25 to $0.48 \mu \mathrm{m}$, whereas the width of the bottom waveguide (WG0) varies from $1.0 \mu \mathrm{m}$ to $0.77 \mu \mathrm{m}$. We use Lumerical finite-difference eigenmode (FDE) solver to calculate the effective refractive indices of $\mathrm{TE}_{0}, \mathrm{TM}_{0}, \mathrm{TE}_{1}$, and $\mathrm{TM}_{1}$ in WG0 and those of $\mathrm{TE}_{0}$ and $\mathrm{TM}_{0}$ in WG1 at the $1.55 \mu \mathrm{m}$ wavelength. As we can see, there is a cross point for $\mathrm{TE}_{1}$ in WG0 and $\mathrm{TE}_{0}$ in $\mathrm{WG}, \mathrm{TM}_{1}$ in WG0 and $\mathrm{TM}_{0}$ in WG1, respectively, which means the input $\mathrm{TE}_{1}$ and $\mathrm{TM}_{1}$ modes in WG0 can adiabatically convert to the $\mathrm{TE}_{0}$ and $\mathrm{TM}_{0}$ modes in the adjacent WG1, respectively. There is no cross point with their own polarization modes for the $\mathrm{TE}_{0}$ and $\mathrm{TM}_{0}$ curves of WG0, which means the input $\mathrm{TE}_{0}$ and $\mathrm{TM}_{0}$ modes will remain propagating in the central waveguide. The width difference for both sides of tapers is chosen as $0.23 \mu \mathrm{m}$ to ensure a large difference of effective indices, where the converted mode would not be coupled back. Thus, in the FMEC, the input $\mathrm{TE}_{0}$ and $\mathrm{TM}_{0}$ modes will be mapped to the central output. The input $\mathrm{TE}_{1}$ and $\mathrm{TM}_{1}$ modes can be converted
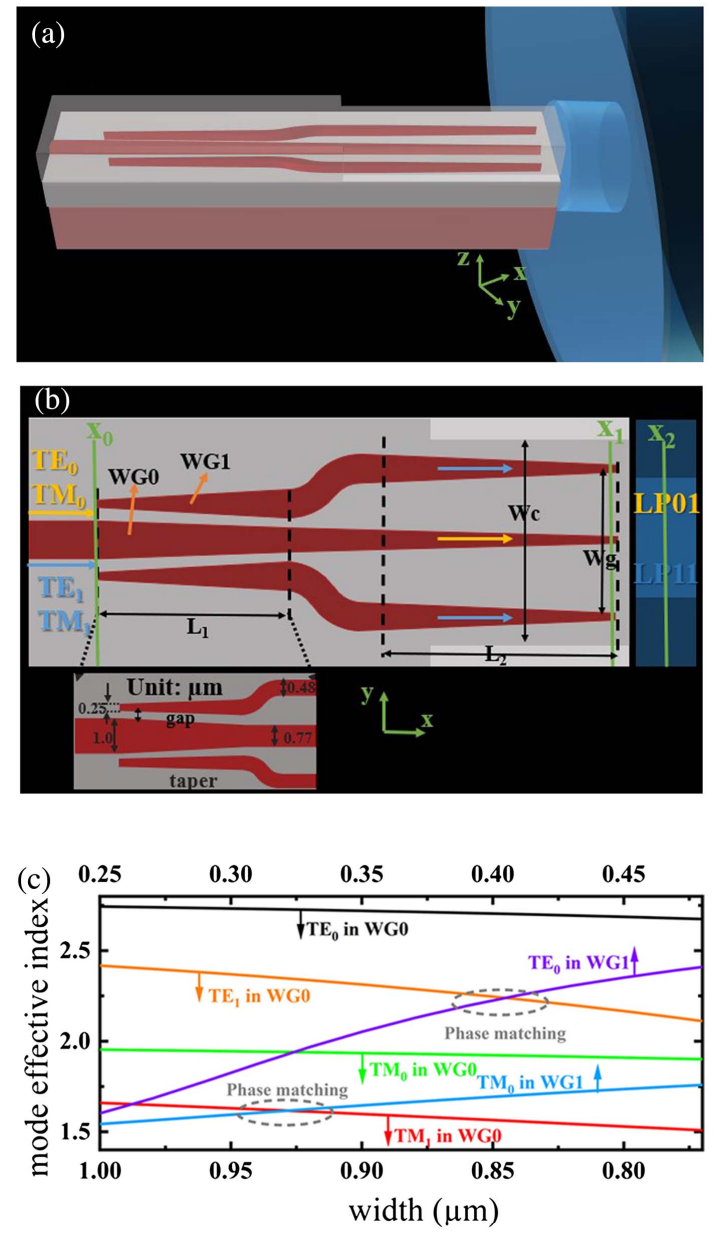

Fig. 1. Schematics of DMEC coupling with dual-mode fiber in (a) 3D view and (b) top view; the silicon waveguides are in red, and the silicon oxide layer is in gray. The modes conversion process is also demonstrated. (c) Effective refractive index of the modes in each waveguide (WGO/WG1) of the modeevolution counter-taper with tapering width from $1 \mu \mathrm{m} / 0.25 \mu \mathrm{m}$ to $0.77 \mu \mathrm{m} / 0.48 \mu \mathrm{m}$.

and divided into two $\mathrm{TE}_{0}$ modes and two $\mathrm{TM}_{0}$ modes with identical intensity and out-phase, and eventually output through the upper and bottom single-mode waveguides of the FMEC separately. For the triple-tip inverse taper, as the waveguide narrows, the middle inverse taper converts the mode field $\left(\mathrm{TE}_{0} / \mathrm{TM}_{0}\right.$ mode) confined in the silicon waveguide into the clad layer, collected by the FMF, and eventually evolves into $\mathrm{LP}_{01, \mathrm{x}}$ and $\mathrm{LP}_{01, \mathrm{y}}$ modes. The mode fields of two side inverse tapers keep expanding between the taper tips and convert into the $\mathrm{TE}_{1} / \mathrm{TM}_{1}$ mode field at the edge of the chip, collected by FMF, and eventually evolve into the $\mathrm{LP}_{11, \mathrm{x}}$ and $\mathrm{LP}_{11, \mathrm{y}}$ modes.

\section{Simulation and Analysis}

\subsection{Optimization of FMEC}

To analyze the performance of FMEC, the simulation is separated into two parts, the on-chip mode conversion from ' $x_{0}$ ' - ' $x_{1}$ ' in Fig. 1(b), and the spatial mode coupling from the 
triple-tip inverse taper to the fiber [' $x_{1}{ }^{\prime}-{ }^{\prime} x_{2}$ ' in Fig. 1(b)]. First, we use the eigenmode expansion (EME) solver to optimize the taper length $\left(L_{1}, L_{2}\right)$, and the parameters used in simulation are shown in Fig. 1(b). In order to satisfy the feature size of commercial silicon foundries, the gaps between the upper and bottom tapers are all set to be $200 \mathrm{~nm}$, and the taper tip width is set to be $100 \mathrm{~nm}$. Figures 2(a) and 2(b) show the losses from the $1 \times 3$ adiabatic mode-evolution counter-taper splitter $\left(L_{1}\right)$ and inverse taper $\left(L_{2}\right)$ at the $1.55 \mu \mathrm{m}$ wavelength. The mode conversion losses are less than $0.2 \mathrm{~dB}$ when $L_{1}>230 \mu \mathrm{m}$, and propagation losses in the inverse taper are less than $0.6 \mathrm{~dB}$ when $L_{2}>400 \mu \mathrm{m}$. Here, we choose $L_{1}=250 \mu \mathrm{m}$ and $L_{2}=450 \mu \mathrm{m}$ to ensure a good trade-off between low loss and small footprint for both modes, and the length of the bending section between the $1 \times 3$ adiabatic mode-evolution counter-taper splitter and inverse taper is $40 \mu \mathrm{m}$, so the whole device has $740 \mu \mathrm{m}$ total length. The on-chip conversion losses of FMEC are less than $0.01 \mathrm{~dB}, 0.02 \mathrm{~dB}, 0.07 \mathrm{~dB}$, and $0.27 \mathrm{~dB}$ for the input $\mathrm{TE}_{0}$, $\mathrm{TM}_{0}, \mathrm{TE}_{1}$, and $\mathrm{TM}_{1}$ modes at the $1.55 \mu \mathrm{m}$ wavelength, respectively, and, in the wavelength range from 1.45 to $1.65 \mu \mathrm{m}$, the mode conversion loss remains lower than $0.62 \mathrm{~dB}$. With the optimized taper length, the transmission spectra of the designed mode-evolution part [' $x_{0}{ }^{\prime}-$ ' $x_{1}$ ' in Fig. 1(b)] are simulated via the 3D-FDTD solver, as shown in Fig. 2(c).

However, the coupling between the triple-tip taper and FMF presents larger coupling loss, mainly caused by the mismatched mode fields between chip edge and FWF. In the simulation model, the diameters of core and cladding of the FMF are $14 \mu \mathrm{m}$ and $125 \mu \mathrm{m}$, with the refractive indices of 1.4485 and 1.44402 , respectively. The silicon substrate may cause more loss because of leaky modes, so we remove the substrate of the SOI wafer ${ }^{[21,22]}$, as shown in Fig. 3. In order to optimize the coupling loss, there are two parts we needed to analyze separately: the integral of mode overlap of the FMF and $\mathrm{SiO}_{2}$ cladding and
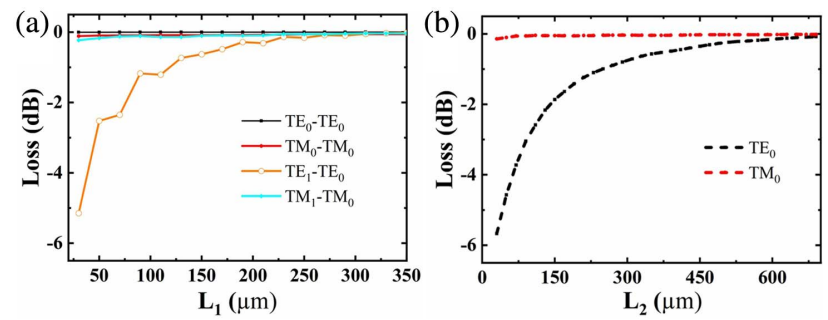

(c)

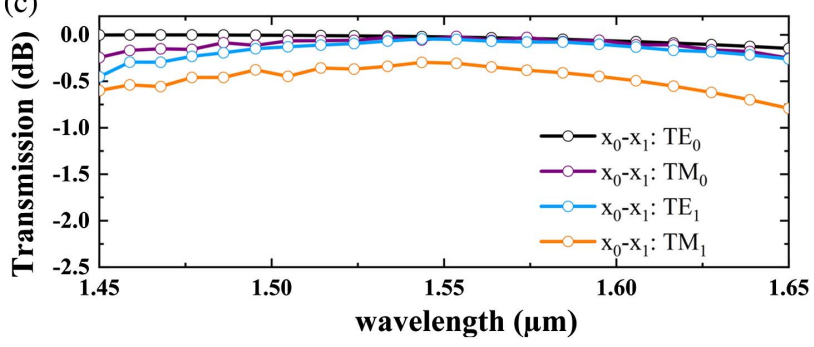

Fig. 2. (a) Mode conversion loss for the input $T E_{0}, T M_{0}, T E_{1}$, and $T M_{1}$ modes, respectively, (b) transmission loss for the $\mathrm{TE}_{0}$ and $\mathrm{TM}_{0}$ modes in inverse taper $\left(L_{2}\right)$, and $(c)$ wavelength dependence of the transmission from ' $x_{0}^{\prime}-{ }^{\prime} x_{1}^{\prime}$. (a)

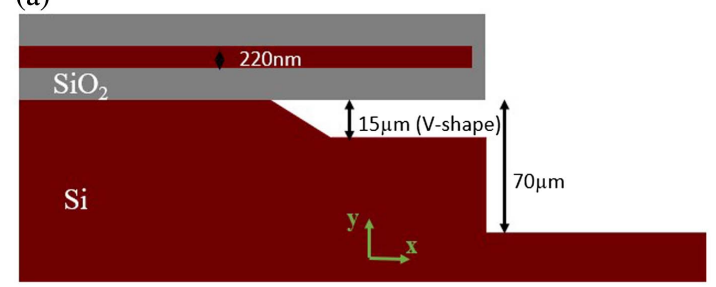

(b)

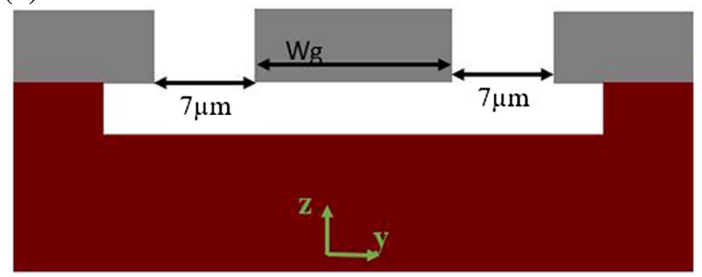

Fig. 3. Cross-sectional schematics of the edge coupling area: (a) $x-y$ direction; (b) $y-z$ direction.

the mode coupling loss between the FMF and silicon tripletip taper. We first optimize the overlap integral in Fig. 4(a). The cladding width (Wc) [Fig. 1(b)] is chosen to be $19 \mu \mathrm{m}$ in order to achieve largest average overlap for four modes. In the second step, we optimize the coupling loss of the silicon triple-tip taper with FMF, which is decided by the taper tip width
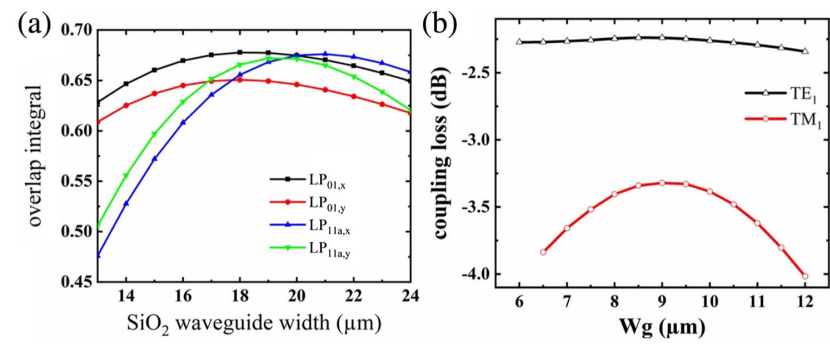

(c)
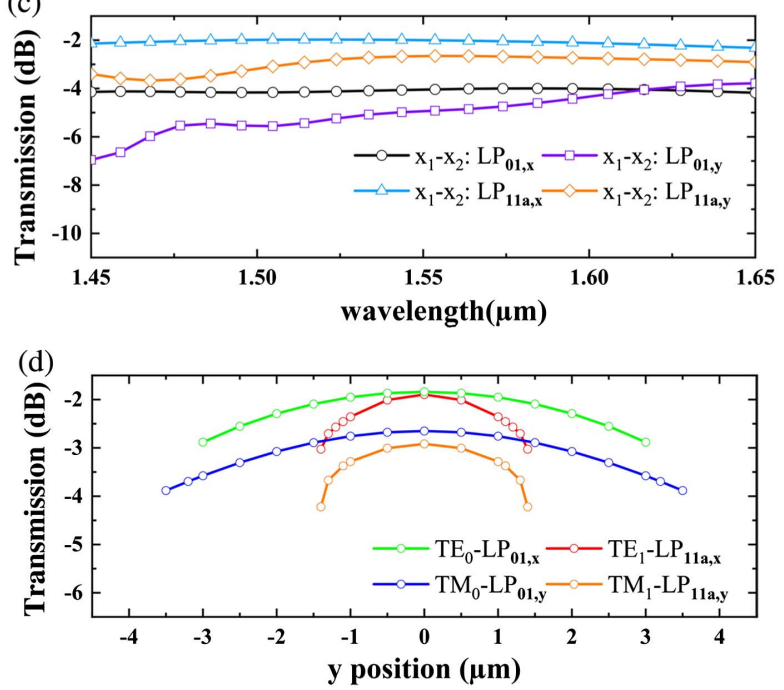

Fig. 4. (a) Simulated overlap integral of mode field between the FMF and $\mathrm{SiO}_{2}$ cladding, (b) simulated coupling loss for different Wg, (c) spatial mode coupling (from ' $X_{1}^{\prime}-{ }^{\prime} X_{2}^{\prime}$ ), and (d) the lateral alignment tolerance of coupling efficiency (CE) for spatial mode coupling. 
and the gap spacing $(\mathrm{Wg})$ between upper and bottom tapers for $\mathrm{TE}_{1}$ and $\mathrm{TM}_{1}$ modes. Figure 4(b) shows the coupling losses for different $\mathrm{Wg}$. The Wg is chosen to be $9 \mu \mathrm{m}$. With the optimized parameters, we calculated the coupling losses between the tripletip taper and FMF in 3D-FDTD, which are $4.0 \mathrm{~dB}, 5.0 \mathrm{~dB}, 2.0 \mathrm{~dB}$, and $2.6 \mathrm{~dB}$ for $\mathrm{TE}_{0}$-to- $\mathrm{LP}_{01, \mathrm{x}}, \mathrm{TM}_{0}$-to- $\mathrm{LP}_{01, \mathrm{y}}, \mathrm{TE}_{1}$-to- $\mathrm{LP}_{11 \mathrm{a}, \mathrm{x}}$, and $\mathrm{TM}_{1}$-to-LP $\mathrm{LP}_{11 \mathrm{a}, \mathrm{y}}$, respectively, as shown in Fig. 4(c) (' $\left.x_{1}{ }^{\prime}-{ }^{\prime} x_{2}{ }^{\prime}\right)$. Considering that those calculation results are based on the case of center excitation, we also simulate the lateral alignment tolerances of spatial mode coupling between the chip edge and FMF. In Fig. 4(d), the simulation results show that the coupling losses of $\mathrm{LP}_{01, \mathrm{x}}, \mathrm{LP}_{01, \mathrm{y}}, \mathrm{LP}_{11 \mathrm{a}, \mathrm{x}}$, and $\mathrm{LP}_{11 \mathrm{a}, \mathrm{y}}$ present the $1 \mathrm{~dB}$ lateral alignment tolerances of $3 \mu \mathrm{m}, 3.2 \mu \mathrm{m}, 1.3 \mu \mathrm{m}$, and $1.3 \mu \mathrm{m}$. Therefore, we need a high accuracy alignment stage to measure the FMEC with high efficiency. Table 1 shows a comparison of the reported state-of-the-art multimode edge coupler. Compared to those types, the proposed FMEC exhibited low loss, more modes, and compatibility with CMOS.

The total coupling efficiency (CE) of the FMEC is $4.1 \mathrm{~dB}$, $5.1 \mathrm{~dB}, 2.1 \mathrm{~dB}$, and $2.9 \mathrm{~dB}$ for $\mathrm{TE}_{0}$-to- $\mathrm{LP}_{01, \mathrm{x}}, \mathrm{TM}_{0}$-to- $\mathrm{LP}_{01, \mathrm{y}}$, $\mathrm{TE}_{1}$-to- $\mathrm{LP}_{11 \mathrm{a}, \mathrm{x}}$, and $\mathrm{TM}_{1}$-to- $\mathrm{LP}_{11 \mathrm{a}, \mathrm{y}}$, respectively, and the crosstalk is less than $-25 \mathrm{~dB}$ with broadband operation, as the spectra of CE and crosstalk shown in Figs. 5(a) and 5(b). As a double-tip inverse taper coupling scheme has been proven for the first highorder mode coupling experimentally ${ }^{[17]}$, we have good potential to realize efficient FMEC with fewer losses experimentally.

Table 1. Comparison of the Reported Multimode Edge Coupler and Coupler Proposed in This Work.

\begin{tabular}{|c|c|c|c|c|}
\hline Ref. & $\begin{array}{l}\text { Number of } \\
\text { Modes }\end{array}$ & $\begin{array}{l}\text { Compatible with } \\
\text { CMOS }\end{array}$ & $\begin{array}{l}\text { Coupling Loss } \\
\qquad(\mathrm{dB})\end{array}$ & $\begin{array}{l}\text { Evaluation } \\
\text { Method }\end{array}$ \\
\hline [10] & 2 & Yes & $\mathrm{TE}_{0}-\mathrm{LP}_{01}: 10.11$ & Numerical \\
\hline 2020 & & & $T E_{1}-L P_{11}: 8.8$ & \\
\hline$[15]$ & 4 & No & $L P_{01}: 8.8$ & Experimental \\
\hline \multirow[t]{3}{*}{2017} & & & $L P_{11 a}: 9.0$ & \\
\hline & & & $L P_{11 b}: 9.6$ & \\
\hline & & & $L P_{21 a}: 9.9$ & \\
\hline [17] & 1 & Yes & $T E_{1}-L P_{11}: 3$ & Numerical \\
\hline 2017 & & & $T E_{1}-L P_{11}: 5.5$ & Experimental \\
\hline [16] & 4 & No & No data & / \\
\hline \multicolumn{5}{|l|}{2020} \\
\hline \multirow[t]{4}{*}{$\begin{array}{l}\text { This } \\
\text { work }\end{array}$} & 4 & Yes & $\mathrm{TE}_{0}-\mathrm{LP}_{01, \mathrm{x}}: 4.1$ & Numerical \\
\hline & & & $\mathrm{TM}_{0}-\mathrm{LP}_{01, y}: 5.1$ & \\
\hline & & & $\mathrm{TE}_{1}-\mathrm{LP}_{11 a, x}: 2.1$ & \\
\hline & & & $T M_{1}-L P_{11 a, y}: 2.9$ & \\
\hline
\end{tabular}
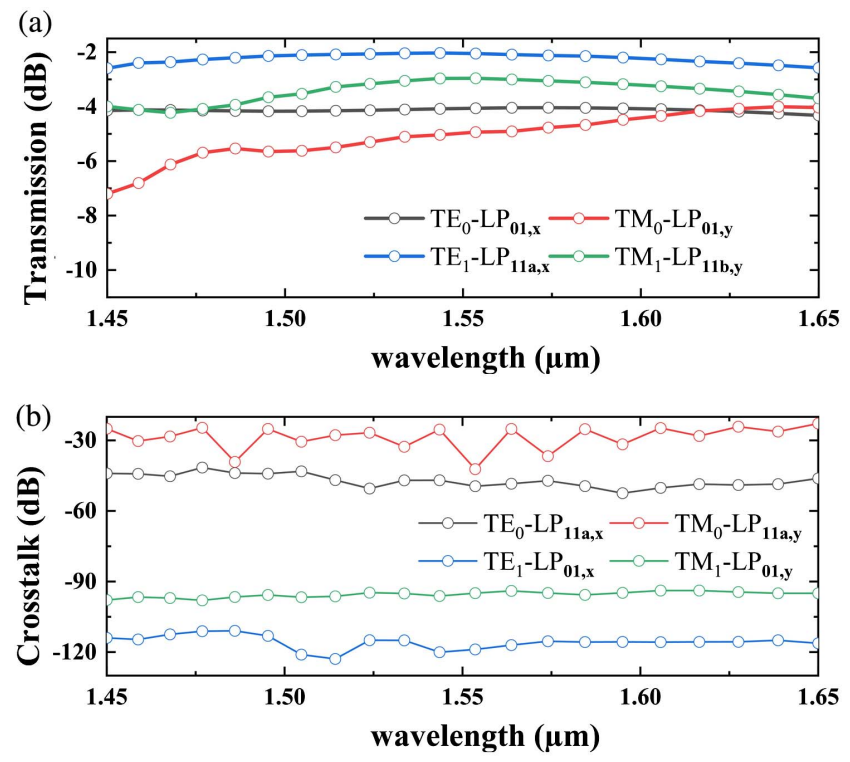

Fig. 5. (a) Total CE and (b) crosstalk of the FMEC in the span of $200 \mathrm{~nm}$.

Figures $6(\mathrm{a})-6(\mathrm{p})$ show the simulated mode propagation for the input $\mathrm{TE}_{0}, \mathrm{TM}_{0}, \mathrm{TE}_{1}$, and $\mathrm{TM}_{1}$ modes, respectively, illustrating the mode conversion and coupling as we expected. Compared to the latest research ${ }^{[10]}$, their simulation results show that the on-chip conversion efficiencies of the dual-mode edge coupler (DMEC) are $69 \%$ and $62 \%$ for $\mathrm{TE}_{0}$ and $\mathrm{TE}_{1}$, respectively, with $3 \mathrm{~dB}$ bandwidth of more than $200 \mathrm{~nm}$. By using a multimode interference (MMI) structure, the large conversion loss enlarged the total coupling loss between the on-chip
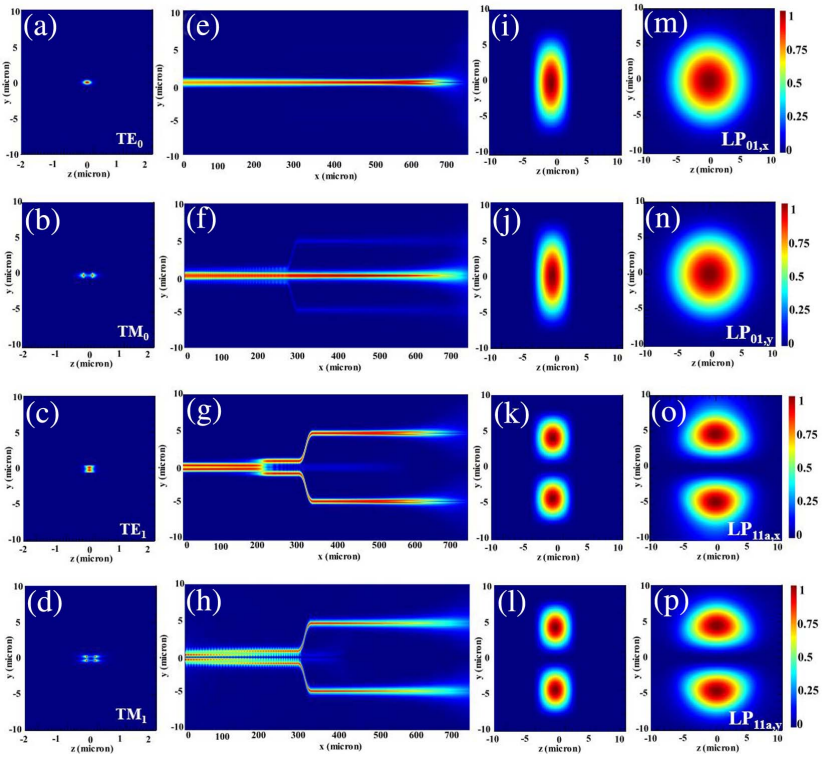

Fig. 6. Simulated electrical field mode profiles of (a) $\mathrm{TE}_{0,}$ (b) $T \mathrm{~T}_{0_{1}}$ (c) $\mathrm{TE}_{1,}$ and (d) $T M_{1}$ modes at position ' $X_{0}^{\prime}$ in Fig. I(b); transmission profiles of the input (e) $T E_{0,}(f) T M_{0,}(g) T E_{11}$ and (h) $T M_{1}$ modes; mode profiles of the (i) $T E_{0,}(j) T M_{0}$ (k) $\mathrm{TE}_{1}$, and (I) $\mathrm{TM}_{1}$ modes in the $\mathrm{SiO}_{2}$ waveguide; mode profiles of (m) $L P_{01, x_{1}}(n) L P_{01, y_{1}}(0) L P_{11 a x_{1}}$ and (p) $L P_{11 a, y}$ modes supported in FMF. 

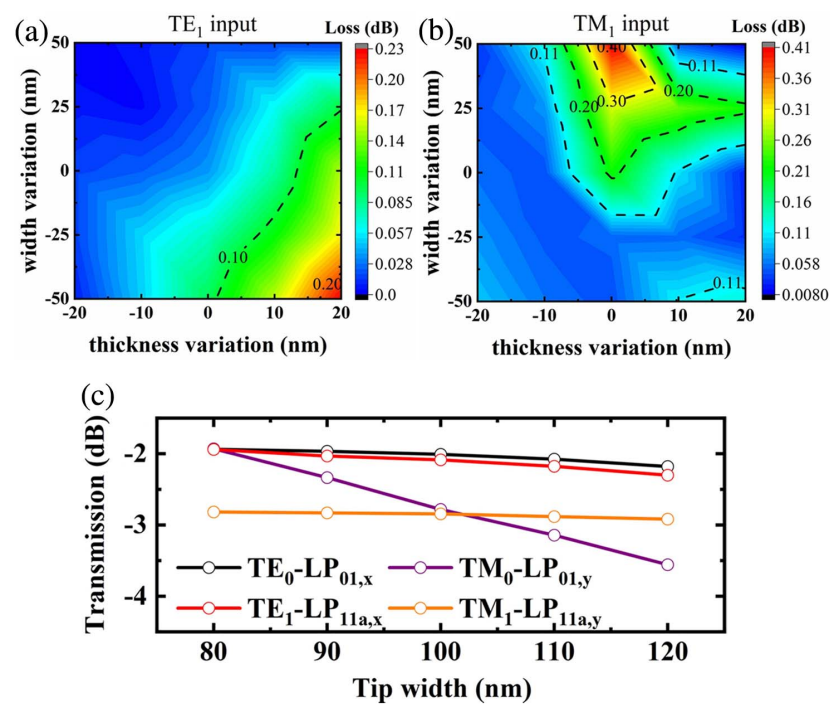

Fig.7. Fabrication tolerance to deviation (a) of the waveguide width and thickness for $\mathrm{TE}_{1}$ input mode, (b) of the waveguide width and thickness for $\mathrm{TM}_{1}$ input mode, (c) of the tip width

multimode waveguide and FWF, resulting in the total coupling loss of DMEC being more than $10 \mathrm{~dB}$ numerically. With our scheme, it can significantly reduce the on-chip conversion loss and scale up to four-mode coupling simultaneously.

\subsection{Analysis of fabrication tolerance}

In order to ensure reliability, Figs. 7(a) and 7(b) show the fabrication tolerance for on-chip conversion using EME by scanning the thickness and tapers waveguide width (which also causes gap distance variations), where the widths of both ends of the tapers vary simultaneously. Compared with that of highorder modes, the loss of input fundamental modes is negligible, so we only consider the conversion loss variations of the input $\mathrm{TE}_{1}$ and $\mathrm{TM}_{1}$ modes under the different fabrication error. The calculated conversion losses are always below $0.42 \mathrm{~dB}$, as long as the waveguide width and thickness are controlled within the fabrication error variations of $\pm 50 \mathrm{~nm}$ and $\pm 20 \mathrm{~nm}$, respectively, which can be readily achieved by commercial silicon photonics foundries. The fabrication tolerance of the mode conversion based on counter-tapers ${ }^{[23]}$ and power splitting based on adiabatical tapers ${ }^{[24,25]}$ have been proved. In addition, the tolerance simulation for the tip width was also performed. Considering $10 \%$ fabrication error, the increase of coupling loss between the inverse taper tip and fiber is below $0.28 \mathrm{~dB}$, as shown in Fig. $7(\mathrm{c})$.

\section{Conclusions}

We design a compact, low loss, broadband, and fabricationtolerant silicon photonic FMEC based on mode-evolution counter-tapers and a triple-tip inverse taper, serving as a bridge between the FMF and multimode chip, so that the input $\mathrm{TE}_{0}$,
$\mathrm{TM}_{0}, \mathrm{TE}_{1}$, and $\mathrm{TM}_{1}$ modes can simultaneously couple into the FMF. The rigorous 3D-FDTD simulations show that the on-chip conversion losses of FMEC are $0.01 \mathrm{~dB}, 0.02 \mathrm{~dB}$, $0.04 \mathrm{~dB}$, and $0.27 \mathrm{~dB}$ for $\mathrm{TE}_{0}, \mathrm{TM}_{0}, \mathrm{TE}_{1}$, and $\mathrm{TM}_{1}$, respectively, and less than $0.62 \mathrm{~dB}$ in the wavelength range from 1.45 to $1.65 \mu \mathrm{m}$, which is negligible for the total coupling loss between FMEC and FMF compared with the coupling loss between triple-tip inverse taper and FMF. The total coupling losses are $4.1 \mathrm{~dB}, 5.1 \mathrm{~dB}, 2.1 \mathrm{~dB}$, and $2.9 \mathrm{~dB}$ for $\mathrm{TE}_{0}$-to- $\mathrm{LP}_{01, \mathrm{x}}$, $\mathrm{TM}_{0}$-to- $\mathrm{LP}_{01, \mathrm{y}}, \mathrm{TE}_{1}$-to- $\mathrm{LP}_{11 \mathrm{a}, \mathrm{x}}$, and $\mathrm{TM}_{1}$-to- $\mathrm{LP}_{11 \mathrm{a}, \mathrm{y}}$, respectively, and less than $7 \mathrm{~dB}$ in the wavelength range from 1.45 to $1.65 \mu \mathrm{m}$ theoretically. Considering fabrication and misalignment errors experimentally ${ }^{[17]}$, we have the potential to realize efficient FMEC with fewer losses experimentally. The device's compatibility with existing silicon photonics foundries and good fabrication tolerance enables the wide usage of the multimode edge coupler on a variety of silicon photonics applications. Considering the conversion loss is small enough for FMEC, further improvements could be adopted by reducing the coupling loss between the triple-tip inverse taper and FMF. A lensed or tapered FWF can be adopted to decrease the mode field area, which can obtain a better agreement with mode field distribution of FMEC. The inverse taper can also realize a better match of the mode field with the FMF and a larger misalignment tolerance through well-designed structures ${ }^{[26-28]}$. Without a complex design and fabrication process, the proposed structure provides much more convenience for both designers and manufacturers. Meanwhile, it also shows good performance under the consideration of fabrication errors. Limited by the silicon thickness of SOI waveguides, this design cannot be applied to vertical higher-order modes, but, combining with different waveguided-core heights, it is also possible to develop more higher-order modes. Further improvements can be done to realize a better performance multimode edge coupler, which can be potentially used to further increase the transmission capability for optical interconnections and communications.

\section{Acknowledgement}

The work was supported by the National Key Research and Development Program of China (No. 2017YFA0206403), Shanghai Municipal Science and Technology Major Project (No. 2017SHZDZX03), National Natural Science Foundation of China (No. 61475180), Science and Technology Commission of Shanghai Municipality (No. 16ZR1442600), Strategic Priority Research Program of Chinese Academy of Sciences (No. XDB24020400), and Shanghai Sailing Program (No. 18YF1428100).

${ }^{\dagger}$ These authors contributed equally to this work.

\section{References}

1. C. Sun, M. T. Wade, Y. Lee, J. S. Orcutt, L. Alloatti, M. S. Georgas, A. S. Waterman, J. M. Shainline, R. R. Avizienis, S. Lin, B. R. Moss, 
R. Kumar, F. Pavanello, A. H. Atabaki, H. M. Cook, A. J. Ou, J. C. Leu, Y. H. Chen, K. Asanovic, R. J. Ram, M. A. Popovic, and V. M. Stojanovic, "Single-chip microprocessor that communicates directly using light," Nature 528, 534 (2015).

2. H. Ma, H. Yang, B. Tang, M. Wei, J. Li, J. Wu, P. Zhang, C. Sun, L. Li, and H. Lin, "Passive devices at $2 \mu \mathrm{m}$ wavelength on $200 \mathrm{~mm}$ CMOS-compatible silicon photonics platform," Chin. Opt. Lett. 19, 071301 (2021).

3. C. Alonso-Ramos, A. Ortega-Monux, I. Molina-Fernandez, P. Cheben, L. Zavargo-Peche, and R. Halir, "Efficient fiber-to-chip grating coupler for micrometric SOI rib waveguides," Opt. Express 18, 15189 (2010).

4. C. Li, D. Liu, and D. Dai, "Multimode silicon photonics," Nanophotonics 8 , 227 (2019).

5. Y. Lai, Y. Yu, S. Fu, J. Xu, P. Shum, and X. Zhang, "Compact double-part grating coupler for higher-order mode coupling," Opt. Lett. 43, 3172 (2018).

6. Z. Yu, Z. Ju, X. Zhang, Z. Meng, F. Yin, and K. Xu, "High-speed multimode fiber imaging system based on conditional generative adversarial network," Chin. Opt. Lett. 19, 081101 (2021).

7. B.-T. Lee and S.-Y. Shin, "Mode-order converter in a multimode waveguide," Opt. Lett. 28, 1660 (2003).

8. Y. Kawaguchi and K. Tsutsumi, "Mode multiplexing and demultiplexing devices using multimode interference couplers," Electron. Lett. 38, 1701 (2002).

9. W. Lu, L. Chang, X. Ren, D. Li, Z. Pan, M. Cheng, D. Liu, and M. Zhang, "Ultra-compact mode (de) multiplexer based on subwavelength asymmetric Y-junction," Opt. Express 26, 8162 (2018).

10. W. Shen, J. Du, J. Xiong, L. Ma, and Z. He, "Silicon-integrated dual-mode fiber-to-chip edge coupler for $2 \times 100 \mathrm{Gbps} / \mathrm{lambda} \mathrm{MOM}$ optical interconnection," Opt. Express 28, 33254 (2020).

11. B. Wohlfeil, G. Rademacher, C. Stamatiadis, K. Voigt, L. Zimmermann, and K. Petermann, "A two-dimensional fiber grating coupler on SOI for mode division multiplexing," IEEE Photon. Technol. Lett. 28, 1241 (2016).

12. A. M. J. Koonen, H. Chen, D. Van, and O. Raz, "Silicon photonic integrated mode multiplexer and demultiplexer," IEEE Photon. Technol. Lett. 24, 1961 (2012).

13. P. C. Kuo, Y. Tong, C. Chow, J. Tsai, Y. Liu, C. Yeh, and H. Tsang, " 4.36 Tbit/s silicon chip-to-chip transmission via few-mode fiber (FMF) using 2D subwavelength grating couplers," in 2021 Optical Fiber Communications Conference and Exhibition (OFC) (2021).

14. Y. Tong, W. Zhou, X. Wu, and H. K. Tsang, "Efficient mode multiplexer for few-mode fibers using integrated silicon-on-insulator waveguide grating coupler," IEEE J. Quantum Electron. 56, 8400107 (2020).
15. Y. Wu and K. Chiang, "Ultra-broadband mode multiplexers based on threedimensional asymmetric waveguide branches," Opt. Lett. 42, 407 (2017).

16. O. A. J. Gordillo, U. D. Dave, and M. Lipson, "Bridging between Si and fewmode fiber higher order modes," in Conference on Lasers and Electro-Optics (Optical Society of America, 2020), paper SM2O.6.

17. Y. Lai, Y. Yu, S. Fu, J. Xu, P. P. Shum, and X. Zhang, "Efficient spot size converter for higher-order mode fiber-chip coupling," Opt. Lett. 42, 3702 (2017).

18. Z. Li, Y. Lai, Y. Yu, and X. Zhang, "Reconfigurable fiber-chip mode converter with efficient multi-mode coupling function," IEEE Photon. Technol. Lett. 32, 371 (2020)

19. A. F. Milton and W. K. Burns, "Tapered velocity couplers for integrated optics: design," Appl. Opt. 14, 1207 (1975).

20. N. Riesen and J. D. Love, "Tapered velocity mode-selective couplers," J. Lightwave Technol. 31, 2163 (2013).

21. J. V. Galán, P. Sanchis, G. Sánchez, and J. Martí, "Polarization insensitive low-loss coupling technique between SOI waveguides and high mode field diameter single-mode fibers," Opt. Express 15, 7058 (2007).

22. M. Wood, P. Sun, and R. M. Reano, "Compact cantilever couplers for lowloss fiber coupling to silicon photonic integrated circuits," Opt. Express 20, 164 (2012)

23. J. Wang, Y. Xuan, M. Qi, H. Huang, Y. Li, M. Li, X. Chen, Z. Sheng, A. Wu, W. Li, X. Wang, S. Zou, and F. Gan, "Broadband and fabrication-tolerant onchip scalable mode-division multiplexing based on mode-evolution countertapered couplers," Opt. Lett. 40, 1956 (2015).

24. V. H. Nguyen, I. K. Kim, and T. J. Seok, "Low-loss and broadband silicon photonic 3-dB power splitter with enhanced coupling of shallow-etched rib waveguides," Appl. Sci. 10, 4507 (2020).

25. J. Zhu, Q. Chao, H. Huang, Y. Zhao, Y. Li, L. Tao, X. She, H. Liao, R. Huang, Z. Zhu, X. Liu, Z. Sheng, and F. Gan, "Compact, broadband, and low-loss silicon photonic arbitrary ratio power splitter using adiabatic taper," Appl. Opt. 60, 413 (2021).

26. Z. Yao, Y. Wan, Y. Zhang, X. Ma, and Z. Zheng, "Broadband high-efficiency triple-tip spot size converter for edge coupling with improved polarization insensitivity," Opt. Commun. 475, 126301 (2020).

27. J. Wang, Y. Xuan, C. Lee, B. Niu, L. Liu, G. Liu, and M. Qi, "Low-loss and misalignment-tolerant fiber-to-chip edge coupler based on double-tip inverse tapers," in 2016 Optical Fiber Communications Conference and Exhibition (OFC) (2016)

28. X. Mu, S. L. Wu, L. R. Cheng, and H. Y. Fu, "Edge couplers in silicon photonic integrated circuits: a review,” Appl. Sci. 10, 29 (2020). 